\title{
Successful Endoscopic Sub Mucosal Resection (ESMR) for Treatment of Carcinoid Tumor with Central Adhesion in Posterior Wall of Duodenum
}

\section{Seyed Reza Fatemi ${ }^{1}$, Naghmeh Salarieh ${ }^{1 *}$, Shabnam Shahrokh ${ }^{1}$, Shirin Haghighi $^{2}$ and Seyed Ali Fatemi ${ }^{3}$}

${ }^{1}$ Gastroenterology and Liver Diseases Research Center, Research Institute for

Gastroenterology and Liver Diseases, Shahid Beheshti University of Medical

Sciences, Tehran, Iran

${ }^{2}$ Departments of Oncology, Shahid Beheshti University of Medical Sciences, Tehran, Iran

${ }^{3}$ Life Science, Biology, student, University of Toronto, Canada

*Corresponding Author: Naghmeh Salarieh, Gastroenterology and Liver Diseases Research Center, Research Institute for Gastroenterology and Liver Diseases, Shahid Beheshti University of Medical Sciences, Tehran, Iran.
Received: May 15, 2021

Published: May 27, 2021

(C) All rights are reserved by Naghmeh

Salarieh., et al.

\begin{abstract}
Duodenal carcinoids are rare form of neuroendocrine tumors with tend to invade the submucosa, during the early stages. Recently, endoscopic resection of neuroendocrine tumors of the duodenum is increasingly performed as an alternative to conventional surgery. Although, a few reports have described the use of endoscopic resection of duodenal carcinoids, it is still a challenging treatment especially for bulbar carcinoids. We performed endoscopic Sub mucosal resection (ESMR) for a case of carcinoid in posterior wall of bulb with central adhesion. Although the tumor was located in the $2^{\text {nd }}$ and submucosa layer, R0 resection was performed without complication in this case. ESMR thus is a safe and effective treatment for duodenal carcinoids less than 10 mm in diameter even with adhesion.
\end{abstract}

Keywords: Endoscopic Sub Mucosal Resection (ESMR); Duodenal Carcinoids; Neuroendocrine Tumors

\section{Introduction}

Duodenal carcinoids are rare form of neuroendocrine tumors with tend to invade the submucosa, during the early stages.

\section{Case Report}

A 49-year-old woman was seen in outpatient clinic because of dyspepsia in the last two years. She had no history of weight loss, nausea, vomiting, fatigue, gastrointestinal bleeding or other alarming signs related to GI system. Her past medical history was not significant except for moderate asthma well controlled with inhalers. Physical examination was unremarkable.
Because of longstanding upper gastrointestinal symptoms (dyspepsia) an upper GI endoscopy was performed and revealed a solitary, sessile submucosal tumor like mass with a depression at the top of lesion in posterior wall of bulb (Figure 1). Biopsy of bulbar lesion consistent with well differentiated neuroendocrine tumor compatible with carcinoid tumor.

Single balloon enteroscopy, small bowel through, colonoscopy and abdominal double contrast CT scan was performed to exclude other sites of disease. No other tumor was found by these diagnostic techniques. 


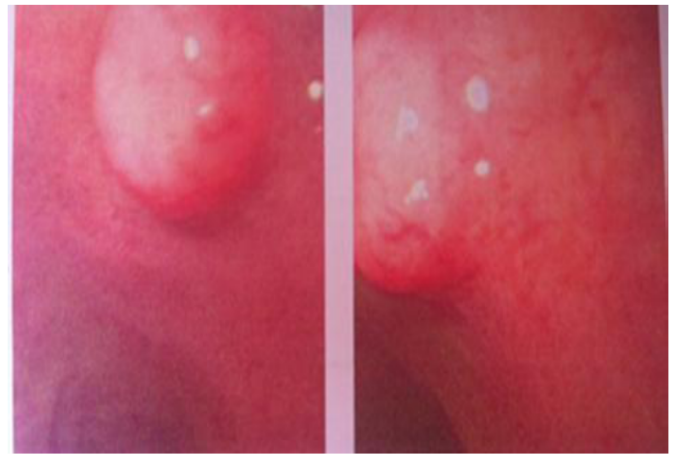

Figure 1: Endo.

Because of the importance of depth of invasion, on outcome and treatment of the lesion, endoscopic ultrasound (EUS) was done (Hitachi 8500 coupled with the EU 3630 radial endoscope, Pentax), which revealed well-defined hypoechoic tumors measuring $7 \mathrm{~mm}$ in diameter confined to the $2^{\text {nd }}$ and $3^{\text {rd }}$ (hyperechoic) SM layer and that the underlying hypoechoic muscularis propria was intact (Figure 2). No lymphadenopathy was seen in region.
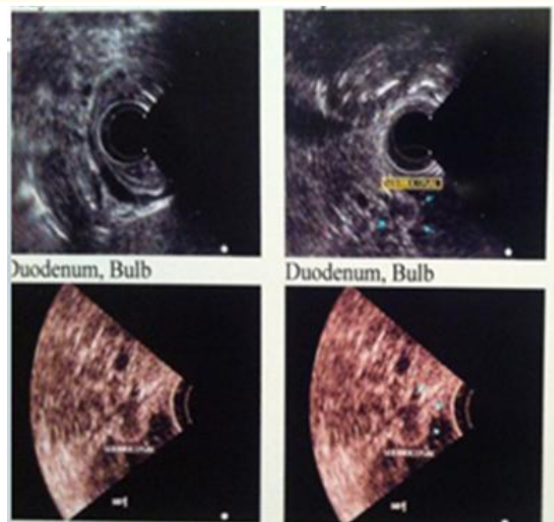

Duodenum, Bulb
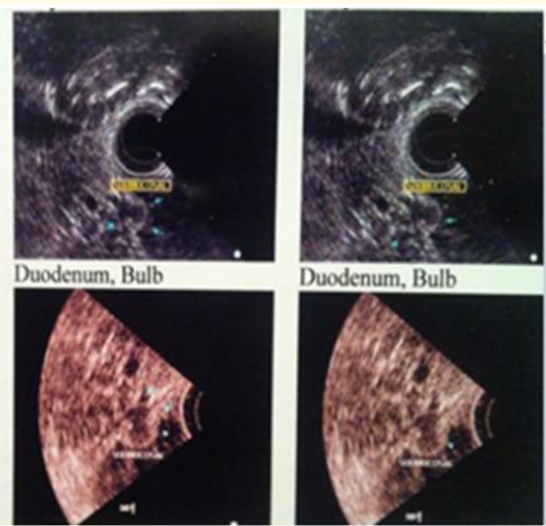

Duodenum, Bulb

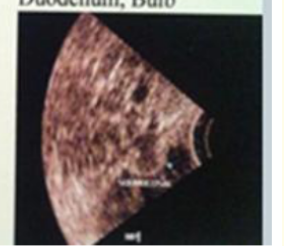

Figure 2: Endosonographic findings of the lesion.

We performed somatostatin receptor scintigraphy (SRS, octreoscan), as a complementary modality to cross sectional imaging to localization of extra abdominal disease site and offers functional information on levels of somatostatin-receptor expression (selec- tion of appropriate candidates with advanced disease for somatostatin-based therapies) which was negative (Figure 3).

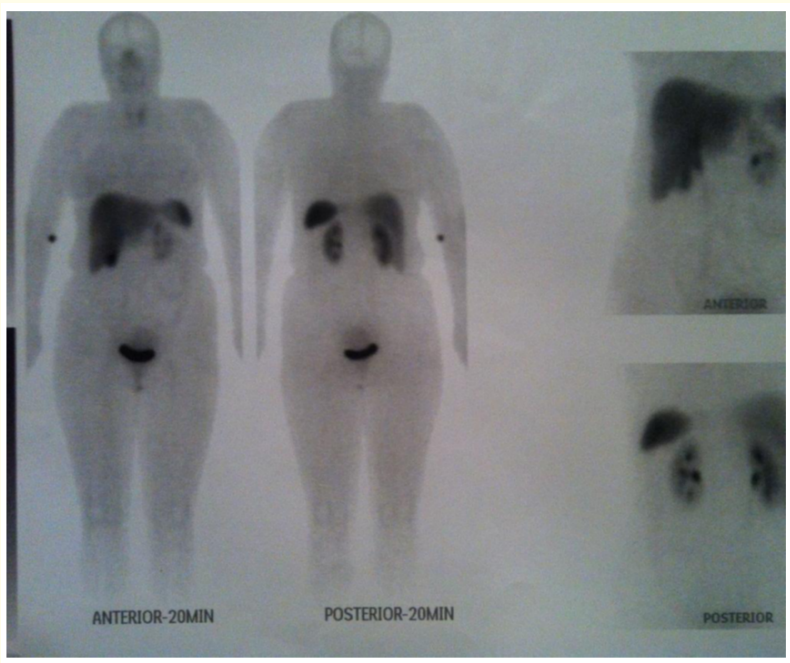

Figure 3

We encountered with nonmetastatic well differentiated of neuroendocrine tumor of small bowel which was limited to submucosal layer.

After obtaining informed consent from the patient, EMR was performed under sedation with a single-channel upper GI endoscope with a water-jet system (GIF-Q260, Olympus). The procedure began with a submucosal injection of normal saline with mixed of $0.01 \%$ epinephrine and $0.4 \%$ indigocarmine dye, to making the cushion. The adequately raised lesion then was snared, using a snare in the same manner as the standard Lift and Cut polypectomy technique. But because of central adhesion, multiple piecemeal resections of whole part of lesion were performed with additional sub mucosal injection of saline and Indigo and it was resected, so Complete resection was performed for the patient without any complication (Figure 4).

To evaluate local recurrence at the resection site, periodic follow-up EGD and EUS was performed for the patient and during 18 months, no evidence of recurrence was detected in pathology and EUS. 


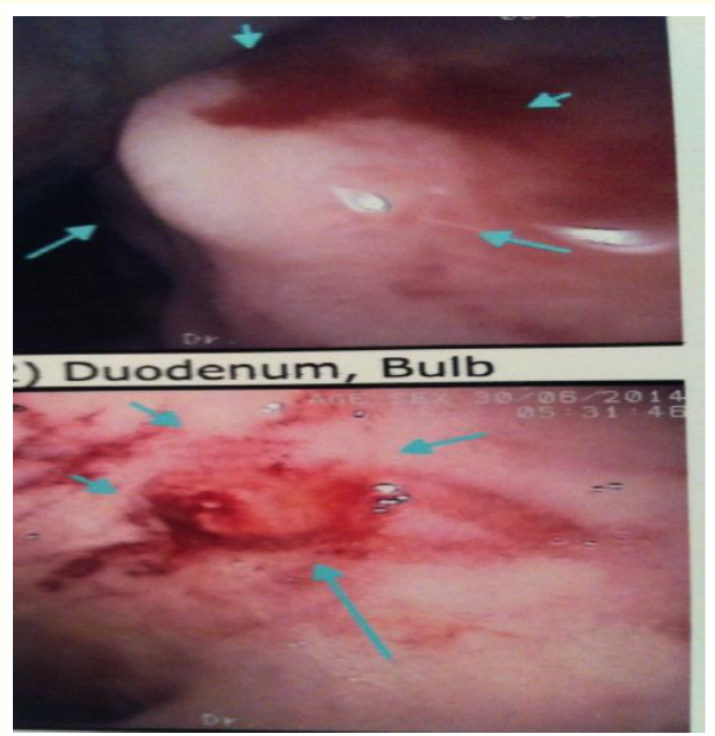

Figure 4

\section{Discussion and Conclusion}

Neuroendocrine tumors (NETs) are a heterogeneous group of neoplasms that derived from various neuroendocrine cell types with different behaviors which can arise widely throughout the body.

The incidence of NETs has been rising over time probably due to increased detection on imaging and endoscopic modalities $[5,6]$ with the annual incidence of clinically significant neuroendocrine tumors approximately 2.5 - 5 per 100,000 and the prevalence has been estimated as 35 per 100,000 [7].

These tumors have different aggressiveness from well-differentiated NETs to Poorly differentiated neuroendocrine carcinomas, which are high-grade carcinomas that resemble small cell carcinoma or large cell neuroendocrine carcinoma of the lung [8] (based on proliferative rate that assessed by mitotic count and/or Ki67 labeling index) [9-11].

NETs of the digestive system (gastroenteropancreatic NETs) are relatively rare with a spectrum of aggressiveness.

Small intestinal neuroendocrine tumors were first distinguished from other tumors in 1907 [12,13]. They were named carcinoid tumors, because their slow growth was considered to be "cancerlike" rather than truly cancerous [13]. Even histologically the majority of these tumors are well-differentiated neuroendocrine tumors that are defined as carcinoid tumors. Other neuroendocrine tumors of the small bowel are rare when compared to carcinoids.

Duodenal neuroendocrine tumors are rare disorders which have benign progression especially when they are small and confined to submucosa [1]. Approximately, $2.6 \%$ of all carcinoid tumors in the United States are reported to be of duodenal carcinoid tumors [2]. Duodenoscopy, computerized tomography, and endoscopic Ultrasound should be performed to evaluate the tumor size, the level of wall invasion, and the presence of regional or distant lymphatic metastases.

Tumor size and involvement of the muscularis propria and the presence of mitotic figures have been proposed as possible risk factors for metastases in duodenal carcinoids [14]. Therefore, the accurate assessment of invasion depth is important for a successful treatment outcome. EUS has been reported to be an appropriate method for assessing carcinoid tumors including duodenal lesions [15-17].

Recently, neuroendocrine EMR of the duodenum Cap Base or Snare Base seems to be a preferable substitute treatment for conventional surgery. Despite of the increasing reports of endoscopic resection of duodenal carcinoids, it is still a challenging treatment. Most reports state that duodenal neuroendocrine tumor can be treated by endoscopic excision, when the diameter is less than 10 mm without periampullary localization or evidence of muscular propria layer invasion assessed by histology and/or endoscopic ultrasound $[3,4]$.

Some recent studies showed that duodenal bulb neuroendocrine, larger than $10 \mathrm{~mm}$ in diameter, can be treated by endoscopic procedure, after confirming the tumor confined to the submucosal layer in EUS examination, and no lymph node involvement by abdominal CT and US [18].

Few methods had been described for EMR of these duodenal lesion such as endoscopic mucosal resection with circumferential mucosal incision (EMR-CMI) [19], using cap technique [17], or the retroflexion during endoscopy [20]. 
Because of limited studies and multiple methods, we reported our case and our technique (ESMR), especially because of lesion with adhesion, our long term follows up revealed that for neuroendocrine tumors less than $1 \mathrm{~cm}$ even in lesion with adhesion to surrounded tissue we could successfully resect them by ESMR method.

\section{Bibliography}

1. Yao JC., et al. "One hundred years after "carcinoid”: epidemiology of and prognostic factors for neuroendocrine tumors in 35,825 cases in the United States". Journal of Clinical Oncology 26 (2008): 3063.

2. Garcia-Carbonero R., et al. "Incidence, patterns of care and prognostic factors for outcome of gastroenteropancreatic neuroendocrine tumors (GEP-NETs): results from the National Cancer Registry of Spain (RGETNE)". Annals of Oncology 21 (2010): 1794.

3. Öberg $\mathrm{K}$ and Castellano D. "Current knowledge on diagnosis and staging of neuroendocrine tumors". Cancer and Metastasis Reviews 30 (2011): 3-7.

4. Gustafsson BI., et al. "Bronchopulmonary neuroendocrine tumors”. Cancer 113 (2008): 5.

5. Travis WD. "The concept of pulmonary neuroendocrine tumors". In: Pathology and Genetics of Tumors of the Lung, Pleura, Thymus and Heart, Travis WD, et al (Eds), IARC Press, Lyon, France (2004): 19.

6. Travis WD., et al. "Survival analysis of 200 pulmonary neuroendocrine tumors with clarification of criteria for atypical carcinoid and its separation from typical carcinoid". The American Journal of Surgical Pathology 22 (1998): 934.

7. Moran CA and Suster S. "Neuroendocrine carcinomas (carcinoid tumor) of the thymus. A clinicopathologic analysis of 80 cases". American Journal of Clinical Pathology 114 (2000): 10.

8. Modlin IM., et al. "Siegfried Oberdorfer: Origins and perspectives of carcinoid tumors". Human Pathology 35.12 (2004): 1440-1451.

9. Arnold R., et al. "Chapter 15 Neuroendocrine Gastro-EnteroPancreatic (GEP) Tumors". In Scheppach W, Bresalier RS, Tytgat GNJ. Gastrointestinal and Liver Tumors". Berlin: Springer (2003): 195-233.
10. Burke AP., et al. "Carcinoid tumors of the duodenum. A clinicopathologic study of 99 cases". Archives of Pathology and Laboratory Medicine 114 (1990): 700-704.

11. Modlin IM., et al. "A 5-decade analysis of 13,715 carcinoid tumors". Cancer 97 (2003): 934-959.

12. Burke AP., et al. "Carcinoid tumors of the duodenum. A clinicopathologic study of 99 cases". Archives of Pathology and Laboratory Medicine 114 (1990): 700-704.

13. Yoshikane H., et al. "Carcinoid tumors of the gastrointestinal tract: evaluation with endoscopic ultrasonography". Gastrointestinal Endoscopy 39 (1993): 375-383.

14. Nishimori I., et al. "Endosonography-guided endoscopic resection of duodenal carcinoid tumor". Endoscopy 29 (1997): 214217.

15. Karagiannis S., et al. "Endoscopic resection with the cap technique of a carcinoid tumor in the duodenal bulb". Endoscopy 41.2 (2009): E288-E289.

16. Dalenback J and Havel G. "Local endoscopic removal of duodenal carcinoid tumors". Endoscopy 36 (2004): 651-655.

17. Jaques Waisberg., et al. The Archives of Gastroenterology 50.1 (2013).

18. Shozo Yokoyama., et al. "Endoscopic resection of duodenal bulb neuroendocrine tumor larger than $10 \mathrm{~mm}$ in diameter.

19. Yuzo Otaki., et al. "Endoscopic mucosal resection with circumferential mucosal incision of duodenal carcinoid tumors". World Journal of Gastrointestinal Endoscopy 5.4 (2013): 197200.

20. Hua LI., et al. "Endoscopic Mucosal Resection of Mucosal Neoplasm Located on the Duodenal Bulb through Endoscope Retroflexion (2010).

\section{Volume 4 Issue 6 June 2021 \\ (C) All rights are reserved by Naghmeh Salarieh., et al.}

